\title{
Über den Alkoholgehalt tierischer Organe.
}

Von

\author{
Georg Landsberg, Arzt.
}

(Aus dem chemischen Laboratorium des pathologischen Instituts zu Berlin.)

(Der Redaktion zugegangen am 19. April 1904.)

Die Frage nach dem Vorkommen von Äthylalkohol in den Organen ist in der medizinischen Literatur schon vielfach erörtert. Der erste, der ihr näher trat, war wohl Hudson Ford ${ }^{1}$ ), der in einer im Jahre 1859 erschienenen Arbeit angab, daß in den Destillaten von Blut sowie von frischen und faulenden Organen sich Alkohol nachweisen lasse. Über ein Jahrzehnt später beschäftigten sich $A$. und J. Béchamp mit dieser Frage. A. Béchamp $\left.{ }^{2}\right)^{3}$ ) zeigte, daß sich in Lebersubstanz, die sofort nach dem Tode durch Eintauchen in Kreosotwasser vor Fäulnis möglichst geschützt war, nach drei bis vier Tagen Alkohol und Essigsäure in beträchtlicher Menge vorfand; die gleichen Bestandteile fand er, ebenfalls in relativ bedeutender Menge, im Destillat frischer Kuh- und Eselinnenmilch. Gleiche Resultate hatte J. Béchamp. ${ }^{4}$ ) In faulendem Fleisch und in frischen Organen wies er Alkohol nach, im frischen Gewebe in kleineren Mengen als bei der Fäulnis. Die Versuche, die Rajewsky ${ }^{5}$ ) über das Vorkommen von Alkohol im Organismus anstellte, führten ihn gleichfalls zu dem Resultate, daß sich in den Organen Alkohol finde. Sein Vorkommen bei der Fäulnis von Eiereiweiß (dextrosehaltigem) stellte Vitali $^{6}$ ) fest. Diese Ansicht, daß Alkohol sich in den Tierorganen vorfinde, blieb jedoch nicht unwidersprochen. Albertoni ${ }^{7}$ ) will ein Vorkommen präformierten oder bei der Fäulnis sich bildenden Alkohols nur für Ausnahmefälle zugeben; ihm schließt sich M. Nicloux $\left.{ }^{8}\right)^{9}$ ) an, der ein Vorkommen von Alkohol in frischen Organen höchstens in Spuren zugeben kann. 
Die Methoden, die für den Nachweis des Alkohols angewendet wurden, waren sehr mannigfach. Im allgemeinen wurden die betreffenden Organe oder Körpersäfte der Destillation unterworfen, in den Destillaten durch mehrfach wiederholte Destillation der Alkohol möglichst konzentriert und im letzten Destillate dann nachgewiesen. Ford bediente sich zum Nachweis der Chromsäurereaktion, der Entzündung des Destillates und einer optischen Erscheinung bei der Destillation, des sogenannten «Spieles» des Alkohols, die sich beim Übergang von Alkohol am Anfang des Kühlrohres in charakteristischer Weise zeigt, sofern die Quantität des Alkohols irgend erheblich ist. A. und J. Béchamp brachten den Nachweis durch Anzünden und durch Oxydation des Alkohols mittels Chromsäure; die quantitative Bestimmung machte A. Béchamp durch Überführung in Essigsäure und Bestimmung der gebildeten Menge Säure durch Titration mit $\mathrm{NaOH}$; J. Béchamp bestimmte die Quantität im letzten Destillate im Sailleronschen Apparate. Der damals vor kurzem angegebenen $\mathrm{CHJ}_{3}$-Reaktion bediente sich Rajew sky; er bezog sie auf Alkohol, weil die Destillate mit Platinmohr Aldehyd bildeten. Daß sich in Organdestillaten stets eine jodoformbildende Substanz vorfindet, gibt auch Albertoni zu. Diese Substanz gibt aber nach Albertoni die Vitalische Reaktion (diese besteht im Auftreten einer weinroten Färbung nach Zusatz von $\mathrm{CS}_{2}+1 \mathrm{gtt} \mathrm{KOH}$ (konz.) + etwas Ammoniummolybdat in Substanz + einige Tropfen verd. $\mathrm{H}_{2} \mathrm{SO}_{4}$ zur Versuchsflüssigkeit) auf Alkohol und Aceton nicht, demnach handelt es sich nicht um Alkohol, sondern wohl um Aldehyd, was auch durch die Jodoformbildung schon in der Kälte wahrscheinlicher ist. Auch im Urin von. Menschen, die keinen Alkohol zu sich genommen hatten, fand schon Lieben ${ }^{11}$ ) stets eine jodoformbildende Substanz, die aber seiner Meinung nach kein Alkohol ist; einen Grund für diese Auffassung führt er nicht an. Gewöhnlich wird im Urin die $\mathrm{CHJ}_{3}$-bildende Substanz auf Aceton bezogen, doch hat Salkowski schon darauf aufmerksam gemacht, daß es sich dabei ebensogut um Aldehyd handeln könne. Da Alkohol, Aldehyd und Aceton die $\mathrm{CHJ}_{3}$-Reaktion geben, so ist es kaum angängig, diese Reaktion mit Bestimmtheit auf eine 
der genannten Substanzen zu beziehen, ohne dafür noch andere Beweismittel anzuführen. Ebenso läßt die Reduktion von $\mathrm{CrO}_{3}$ $\mathrm{zu} \mathrm{Cr}_{2} \mathrm{O}_{3}$ und der damit eintretende Farbenwechsel von Gelb in Grün nichts anderes schließen, als daß reduzierende Körper in der untersuchten Flüssigkeit sind. Diese Reduktion kommt nicht dem Alkohol allein zu, sondern in gleicher Weise auch dem Aldehyd; nach der Angabe von Bodländer, ${ }^{10}$ ) die ich bestätigen kann, wirkt sogar Essigsäure unter bestimmten Versuchsbedingungen noch reduzierend. $\mathrm{Zu}$ berücksichtigen ist schließlich noch der Umstand, daß die $\mathrm{CHJ}_{3}$-bildenden bezw. $\mathrm{CrO}_{3}$-reduzierenden Körper nicht präformiert in den Organen enthalten zu sein brauchen, sondern auch erst bei der Destillation entstanden sein können.

Der reduzierenden Wirkung, die Alkohol auf $\mathrm{CrO}_{3}$ ausübt, bediente sich M. Nicloux zur Ausarbeitung einer quantitativen Bestimmungsmethode kleiner Mengen Alkohols. Seiner Angabe nach war er der erste, der eine solche Methode angab, vor ihm soll eine chemische Methode zur quantitativen Alkoholbestimmung nicht bestanden haben. Nach Nicloux werden $\mathrm{zu}$ einer bestimmten kleinen Menge (5 ccm) der zu untersuchenden Flüssigkeit im Reagensglase einige Kubikzentimeter konzentrierter Schwefelsäure hinzugefügt, die Mischung bis zum Sieden erhitzt und dann aus einer Bürette solange eine $\mathrm{K}_{2} \mathrm{Cr}_{2} \mathrm{O}_{7}-$ Lösung von bestimmtem Gehalt zugesetzt, bis die anfangs auftretende grünblaue Farbe der Flüssigkeit in eine grüngelbe übergeht. Da die Endreaktion nicht sehr scharf ist, so rät er, für bestimmte Verdünnungen von Alkohol (zwischen $1 / 500$ und $1 / 3000$ ) je zwei Reagensgläschen herzustellen, von denen in dem einen zu der schwefelsauren Alkohollösung nur soviel Chromlösung hinzugefügt wird, daß es noch kein $\mathrm{CrO}_{3}$ im Überschuß enthält, während in dem zweiten durch Zusatz von ein bis zwei Tropfen mehr schon ein Überschuß vorhanden ist. Durch Vergleich mit diesen Proben soll die Erkennung der Endreaktion besser möglich werden. Auch soll man der ersten Titration eine zweite folgen lassen, bei der man sogleich die zuerst gefundene Menge von $\mathrm{K}_{2} \mathrm{Cr}_{2} \mathrm{O}_{7}$-Lösung zusetzt und sieht, ob der Farbenausschlag auch hier schon eintritt; sonst muß 
man jetzt eventuell noch einige Tropfen der Chromlösung hinzufügen. Was den Verlauf der Reaktion betrifft, so soll der Alkohol dabei bis auf $2 \%$ zu Essigsäure werden.

Die Nachprüfung von Niclouxs Angaben ergab folgendes:

Lösung von $1 \mathrm{ccm} \mathrm{Alk.} \mathrm{absol.:} 1000 \mathrm{Aq}$. dest., $5 \mathrm{ccm}$ der Lösung $+3 \mathrm{ccm}$ konzentrierte $\mathrm{H}_{2} \mathrm{SO}_{4}$ zum Sieden erhitzt, darauf $\mathrm{K}_{2} \mathrm{Cr}_{2} \mathrm{O}_{7}$-Lösung (19 g : 1 l Aq. dest.) zugesetzt. Der Farbenumschlag tritt ein bei $0,8 \mathrm{ccm} ; 0,8 \mathrm{ccm} ; 0,9 \mathrm{ccm} ; 0,8 \mathrm{ccm}$; $0,9 \mathrm{ccm} ; 0,7 \mathrm{ccm} ; 0,8 \mathrm{ccm}$ etc. Sehr zahlreiche Titrationen zeigen, daß im Durchschnitt 0,83 ccm der Cr-Lösung verbraucht werden. Dieselbe Alkohollösung, mit einer $\mathrm{K}_{2} \mathrm{Cr}_{2} \mathrm{O}_{7}$-Lösung von $1 / 4$ der Stärke der vorigen titriert, zeigt den Farbenumschlag bei $3,0 \mathrm{ccm} ; 3,3 \mathrm{ccm} ; 2,8 \mathrm{ccm} ; 3,4 \mathrm{ccm} ; 3,2 \mathrm{ccm} ; 3,3 \mathrm{ccm}$; 3,2 ccm etc., im Durchschnitt also bei $3,26 \mathrm{ccm}$.

Lösung von $1 \mathrm{ccm}$ Alk. absol. : 2000 Aq. dest. Die gleiche Menge Alkohollösung, in gleicher Weise behandelt, ergibt bei Zusatz der weniger konzentrierten $\mathrm{K}_{2} \mathrm{Cr}_{2} \mathrm{O}_{7}$-Lösung $(4,75 \mathrm{~g}$ im Liter) den Farbenumschlag bei $1,6 \mathrm{ccm} ; 1,5 \mathrm{ccm} ; 1,8 \mathrm{ccm}$; $1,6 \mathrm{ccm} ; 1,7 \mathrm{ccm} ; 1,6 \mathrm{ccm}$ etc., im Durchschnitt bei $\mathbf{1 , 6 4} \mathrm{ccm}$ Zusatz.

Lösung von $1 \mathrm{ccm}$ Alk. absol. : 4000 Aq. dest; gleiche Menge in gleicher Weise behandelt; ergibt bei Zusatz der CrLösung Farbenumschlag bei $0,8 \mathrm{ccm} ; 0,6 \mathrm{ccm} ; 0,6 \mathrm{ccm} ; 0,7 \mathrm{ccm}$ etc., im Durchschnitt bei $0,74 \mathrm{ccm}$ Zusatz.

Es ergibt sich also, daß die Endreaktion schon früher eintritt, als Nicloux angibt, daß für die Oxydation von $0,005 \mathrm{ccm}$ Alkohol nicht $1 \mathrm{ccm}$ hezw. $4 \mathrm{ccm} \mathrm{K}_{2} \mathrm{Cr}_{2} \mathrm{O}_{7}$-Lösung verbraucht werden, sondern nur 0,83 bezw. $3,26 \mathrm{ccm}$. Setzt man, wie Nicloux in späteren Arbeiten vorschlägt, zu $5 \mathrm{ccm}$ der Alkohollösung 6-7 ccm konzentrierte $\mathrm{H}_{2} \mathrm{SO}_{4}$ hinzu, so nähern sich die Zahlen allerdings den von Nicloux angegebenen und betragen $\mathbf{0 , 9 1}$ bezw. $\mathbf{3 , 6} \mathrm{ccm}$. Diesen letzten Zahlen entsprechen ungefähr die Zahlen, die Béhal und François ${ }^{12}$ ) in einer Nachprüfung der Niclouxschen Angaben gefunden haben. Sie verwenden nämlich eine Lösung von $16,97 \mathrm{~g}$ $\mathrm{K}_{2} \mathrm{Cr}_{2} \mathrm{O}_{7}$ im Liter, wovon $1 \mathrm{ccm}=0,005 \mathrm{ccm}$ Alk. absol. entsprechen soll. 
Man ersieht aus den Zahlen der Nachprüfung, daß die Titration keine genaue ist, daß bei Zusatz verschiedener Mengen Schwefelsäure auch die Menge der zuzusetzenden $\mathrm{K}_{2} \mathrm{Cr}_{2} \mathrm{O}_{7}$ Lösung variiert. Dies erklärt sich wahrscheinlich daraus, daß in stärker saurer Lösung auch die Oxydation eine weitergehende ist. Die Niclouxsche Angabe nämlich, daß der Alkohol quantitativ $\mathrm{zu}$ Essigsäure ( $2 \%$ sogar bis $\mathrm{zu} \mathrm{CO}_{z}$ ) oxydiert wird, ist sicherlich unrichtig; man kann sich vielmehr davon überzeugen, daß bei Ausführung der Bestimmung stets ein intensiver Aldehydgeruch auftritt, ein Teil des oxydierten Alkohols somit als Aldehyd entweicht und weiterer Oxydation entgeht. Es ist auch nicht ausgeschlossen, daß beim Erhitzen der schwefelsauren Alkohollösung schon etwas vom Alkohol unoxydiert verdunstet. Aus der Verschiedenheit dieser Faktoren erklären sich somit nach meiner Auffassung die Differenzen der Zahlen bei den einzelnen Titrationen, soweit sie nicht durch den nicht sehr scharfen Farbenumschlag bedingt sind. Immerhin aber kann man wohl sagen, daß die Titrationsmethode rein empirisch ganz brauchbar ist, wobei selbstverständlich nicht nochmals hervorgehoben $\mathrm{zu}$ werden braucht, daß der Aldehyd z. B. in gleicher Weise reduzierend wirkt.

Ein Punkt aber verdient noch besonderer Erwähnung, der nämlich, daß Nicloux als erster die angegebene Methode zur quantitativen Alkoholbestimmung benutzt haben will. Gewiß muß man annehmen, daß ihm die Arbeiten über diesen Gegenstand unbekannt gewesen sind und scheinbar noch sind; indes wurde eine Methode, die der Niclouxschen im Prinzip vollständig analog ist, schon vor über zwanzig Jahren von Bodländer ${ }^{10}$ ) verwendet. $O b$ diese Methode von Bodländer stammt, ist aus seiner Arbeit nicht ersichtlich. Bei ihr wird $\mathrm{zu}$ einer Lösung von $\mathrm{CrO}_{3}$ in konzentrierter $\mathrm{H}_{2} \mathrm{SO}_{4}(1 \mathrm{~g}$ auf $300 \mathrm{ccm} \mathrm{H}_{2} \mathrm{SO}_{4}$ ) so lange von der zu untersuchenden Alkohollösung hinzugesetzt, bis die ursprünglich gelbe Farbe in ein tiefes Grün übergegangen ist. Auch diese Methode leidet an dem gleichen Fehler wie die Niclouxsche, an der wenig scharfen Endreaktion; bei ihr ist die Oxydation eine viel weitergehende, nach Bodländer in der Hauptsache bis zur $\mathrm{CO}_{2}$, 
und es wird daher auch für die gleiche Menge Alkohol weit mehr $\mathrm{CrO}_{3}$ gebraucht. Indes hat sie den Vorzug der größeren Bequemlichkeit, da das Arbeiten in der Hitze fortfällt, vielleicht werden dadurch auch manche Ungenauigkeiten der Niclouxschen Methode vermieden. Auch als kolorimetrische Methode wurde diese Methode von Straßmann ${ }^{13}$ ) verwendet. Daß sie im sonstigen Ausland nicht ganz unbekannt war, zeigt eine Arbeit von Benedict und Norris ${ }^{14}$ ) aus dem Jahre 1898, welche eine Modifizierung der Bodländerschen Methode vorschlägt, um das Entweichen von Aldehyd zu vermeiden. Da die Bodländersche Methode erst im Verlaufe dieser Arbeit zu meiner Kenntnis kam, verwendete ich die Niclouxsche zur quantitativen Alkoholbestimmung.

Um den Alkohol qualitativ möglichst einfach und sicher nachzuweisen, wurde folgender Weg eingeschlagen. Es wurde die zu untersuchende Flüssigkeit mit $\mathrm{H}_{2} \mathrm{SO}_{4}$ und $\mathrm{K}_{2} \mathrm{CrO}_{4}$ bezw. äquivalenten Mengen $\mathrm{K}_{2} \mathrm{Cr}_{2} \mathrm{O}_{7}$ in bestimmtem Verhältnis versetzt, destilliert und das Destillat in Fraktionen aufgefangen. Es sollte dabei der Alkohol in Aldehyd übergeführt und als solcher nachgewiesen werden. Da Aldehyd schon bei $21^{\circ} \mathrm{C}$. siedet, so mußte der Aldehyd in seiner Hauptmenge in die ersten Kubikzentimeter des Destillates übergehen. Dies war denn auch stets der Fall, wenn auch das vierte Destillat oft noch deutliche Aldehydreaktionen aufwies. Zum Nachweis des Aldehyds dienten folgende Reaktionen:

1. Die Bildung von Aldehydharz durch Erhitzen mit konzentrierter $\mathrm{NaOH}$. Diese Reaktion wird in reiner Aldehydlösung (Aldehyd conzentr. von Schering) mit Bildung eines Harzniederschlages bis zur Konzentration von 1:6000 gegeben; bei einer Konzentration von 1:10000 tritt nur noch ganz schwache Gelbfärbung auf. Daneben ist für die Reaktion ein Geruch charakteristisch, der an den Geruch geschmorter Birnen erinnert.

2. Die Reduktion von ammoniakalisch-alkalischer Silberlösung und Bildung eines Silberspiegels. Diese Reaktion ist sehr fein, doch muß man sich durch Kontrollversuche davon überzeugen, daß das Reagens nicht allein schon reduziert wird, 
was wohl durch den oftmaligen Gehalt des $\mathrm{NH}_{3}$ an organischen Substanzen bedingt wird.

3. Die Reaktion mit Nesslers Reagens nach Crismer, bei der bei Verdünnungen von 1:25000 noch ein schwacher Niederschlag, von 1 : 100000 noch deutliche Gelbfärbung auftritt.

4. Die Jodoformprobe nach Lieben, die ebenfalls in Konzentrationen von $1: 100000$ noch deutlichen Jodoformgeruch und nach längerem Stehen Ausscheidung von Jodoformkristallen darbietet.

Es handelte sich nun darum, wie groß man den Zusatz von $\mathrm{H}_{2} \mathrm{SO}_{4}$ und von $\mathrm{K}_{2} \mathrm{CrO}_{4}$ wählen sollte, um die Oxydation des Alkohols bis zum Aldehyd und nicht darüber hinaus zu führen. $\mathrm{Zu}$ diesem $\mathrm{Zweck}$ wurden folgende Versuche ausgeführt. Es wurden stets $100 \mathrm{ccm}$ verschieden starker Alkohollösung mit verschiedenen Mengen $\mathrm{H}_{2} \mathrm{SO}_{4}$ bezw. $\mathrm{K}_{2} \mathrm{CrO}_{4}$ versetzt und destilliert. Es wurden 2 oder 3 Destillate à $15 \mathrm{ccm}$ aufgefangen und die Intensität der Aldehyudreaktionen in diesen Destillaten verglichen. Die Einzelheiten ergeben sich aus umstehender Tabelle.

Aus der Tabelle ist ersichtlich, daß der Zusatz verschiedener Mengen von $\mathrm{H}_{2} \mathrm{SO}_{4}$ zur Destillation auf die Intensität der Reaktionen von erheblichem Einfluß ist. Die stärksten Reaktionen fanden sich bei Zusatz von $5 \mathrm{ccm}$ konzentrierter $\mathrm{H}_{2} \mathrm{SO}_{4} \mathrm{zu}$ $100 \mathrm{ccm}$ der Alkohollösung, wobei abwärts von einer Konzentration von 1:5000 diese keine Rolle spielte; ebenso erwies sich als am zweckmäßigsten der Zusatz von $5 \mathrm{ccm}$ einer $2 \%$ igen $\mathrm{K}_{2} \mathrm{CrO}_{4}$-Lösung. Bei Zusatz von mehr $\mathrm{H}_{8} \mathrm{SO}_{4}$ oder $\mathrm{K}_{2} \mathrm{CrO}_{4}$ war eine Zunahme der Intensität der Reaktionen nicht $\mathrm{zu}$ bemerken; in einzelnen Fällen sogar eine deutliche Abnahme. Es wurde daher bei der Behandlung der Organdestillate die hier als zweckmäßig gefundene Menge $\mathrm{H}_{2} \mathrm{SO}_{4}$ und $\mathrm{K}_{2} \mathrm{CrO}_{4}$-Lösung zugesetzt. Aus der Tabelle ergibt sich ferner, daß bis $\mathrm{zu}$ einem Gehalt von $0,02 \mathrm{ccm}$ Alkohol abs. der destillierten Lösung die Reaktion mit $\mathrm{NaOH}$ im 1. Destillat stark positiv ausfiel; ihre Grenze lag, wie andere Versuche zeigten, bei einem Gehalt von $0,014 \mathrm{ccm}$ Alkohol abs., entsprechend $100 \mathrm{ccm}$ einer Lösung 1:7000. 


\begin{tabular}{|c|c|c|c|c|c|c|}
\hline & \begin{tabular}{|} 
Menge \\
und Kon- \\
zentration \\
der \\
Lösung
\end{tabular} & $\begin{array}{c}\text { Zusalz } \\
\text { bei der Destillation }\end{array}$ & \begin{tabular}{|c|} 
Zahl \\
der \\
Destil- \\
late \\
$\grave{a}$ \\
$10 \mathrm{ccm}$ \\
\end{tabular} & $\begin{array}{c}\text { I. } \\
\text { Aldehydharz- } \\
\text { Bildung }\end{array}$ & $\begin{array}{c}\text { Ausfall der } \\
\text { II. } \\
\mathrm{CH}_{3^{-}} \\
\text {Bildung } \\
\end{array}$ & $\begin{array}{l}\text { Reaktionen im } \\
\begin{array}{|c|}\text { III. } \\
\text { Nesslers } \\
\text { Reagens }\end{array}\end{array}$ \\
\hline 1 & $\begin{array}{l}100 \mathrm{ccm} \\
1: 1000\end{array}$ & $\begin{array}{l}5 \mathrm{ccm} \mathrm{H}_{2} \mathrm{SO}_{4} \\
2,5, \mathrm{~K}_{2} \mathrm{CrO}_{4}\end{array}$ & 3 & \begin{tabular}{|c|} 
s. schwacher \\
gelb. Niederschl.
\end{tabular} & \begin{tabular}{|l|} 
kalt: mäßiger \\
Niederschlag \\
\end{tabular} & $\begin{array}{c}\text { starker } \\
\text { Niederschlag }\end{array}$ \\
\hline 2 & $\begin{array}{l}100 \mathrm{ccm} \\
1: 1000\end{array}$ & 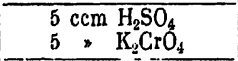 & 3 & $\begin{array}{c}\text { mäßjger, gelher } \\
\text { Niederschlag } \\
\end{array}$ & $\begin{array}{l}\text { sehr starker } \\
\text { Niederschlag }\end{array}$ & $\begin{array}{c}\text { starker, balld } \\
\text { graner Siedersectlag }\end{array}$ \\
\hline 3 & $\begin{array}{l}100 \mathrm{ccm} \\
1: 1000\end{array}$ & $\begin{array}{c}5 \mathrm{ccm} \mathrm{H}_{2} \mathrm{SO}_{4} \\
10 \gg \mathrm{K}_{2} \mathrm{CrO}_{4}\end{array}$ & 2 & $\begin{array}{c}\text { starker, gelber } \\
\text { Niederschlag } \\
\end{array}$ & do. & do. \\
\hline 4 & $\begin{array}{c}100 \mathrm{ccm} \\
1: 1000 \\
\end{array}$ & $\begin{array}{c}5 \mathrm{ccm} \mathrm{H}_{2} \mathrm{SO}_{4} \\
15 \geqslant \mathrm{~K}_{2} \mathrm{CrO}_{4} \\
\end{array}$ & 2 & do. & do. & do. \\
\hline 5 & $\begin{array}{l}100 \mathrm{ccm} \\
1: 2000\end{array}$ & 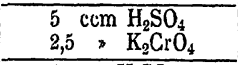 & 2 & $\begin{array}{c}\text { mäfiger, gelber } \\
\text { Niederschlag }\end{array}$ & do. & do. \\
\hline 6 & $\begin{array}{c}100 \mathrm{ccm} \\
1: 2000\end{array}$ & $\begin{array}{l}5 \mathrm{ccm} \mathrm{H}_{2} \mathrm{SO}_{4} \\
5 \gg \mathrm{K}_{2} \mathrm{CrrO}_{4}\end{array}$ & 2 & $\begin{array}{l}\text { starker, gelber } \\
\text { Niederschlag }\end{array}$ & do. & do. \\
\hline 7 & $\begin{array}{l}100 \mathrm{ccm} \\
1: 5000\end{array}$ & $\begin{array}{l}11 \mathrm{~cm} \mathrm{H}_{2} \mathrm{SO}_{4} \text { verdünnt } \\
5 \gg \mathrm{K}_{2} \mathrm{CrO}_{4}\end{array}$ & 2 & negativ & $\begin{array}{c}\text { mäßiger } \\
\text { Niederschlag }\end{array}$ & $\begin{array}{c}\text { mäßiger, gelber } \\
\text { Niederschlag }\end{array}$ \\
\hline 8 & $\begin{array}{c}100 \mathrm{ccm} \\
1: 5000 \\
\end{array}$ & $\begin{array}{l}3 \mathrm{ccm} \mathrm{H}_{2} \mathrm{SO}_{4} \text { rerdünnt } \\
5 * \mathrm{~K}_{2} \mathrm{CrO}_{4} \\
\end{array}$ & 2 & do. & $\begin{array}{c}\text { starker } \\
\text { Niederschlag } \\
\end{array}$ & do. \\
\hline 9 & $\begin{array}{c}100 \mathrm{ccm} \\
1: 5000 \\
\end{array}$ & $\begin{array}{l}5 \mathrm{ccm} \mathrm{H}_{2} \mathrm{SO}_{4} \text { verdünnt } \\
2,5 * \mathrm{~K}_{2} \mathrm{Cr} \mathrm{rO}_{4}\end{array}$ & 3 & $\begin{array}{c}\text { schwache } \\
\text { Gelbfärbung }\end{array}$ & do: & $\begin{array}{l}\text { starker, gelber } \\
\text { Niederschlag } \\
\end{array}$ \\
\hline 10 & $\begin{array}{c}100 \mathrm{ccm} \\
1: 5000\end{array}$ & 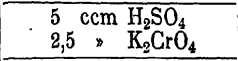 & 3 & $\begin{array}{l}\text { geringer, gelber } \\
\text { Niederschlag } \\
\end{array}$ & $\begin{array}{l}\text { sehr starker } \\
\text { Niederschlag } \\
\end{array}$ & do. \\
\hline 11 & $\begin{array}{c}100 \mathrm{ccm} \\
1: 5000 \\
\end{array}$ & 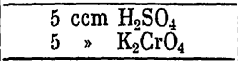 & 2 & $\begin{array}{l}\text { mäßiger, gelber } \\
\text { Niederschlag }\end{array}$ & do. & $\begin{array}{l}\text { do., bald grau } \\
\text { werdend }\end{array}$ \\
\hline 12 & $\begin{array}{c}100 \mathrm{ccm} \\
1: 5000\end{array}$ & 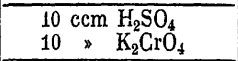 & 2 & $\begin{array}{l}\text { geringer, gelber } \\
\text { Niederschlag }\end{array}$ & do. & do. \\
\hline 13 & $\begin{array}{l}100 \mathrm{ccm} \\
1: 7000\end{array}$ & 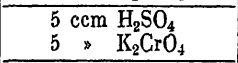 & 2 & $\begin{array}{c}\text { schwache } \\
\text { Gelbfärbung }\end{array}$ & do. & do. \\
\hline 14 & $\begin{array}{l}100 \mathrm{ccm} \\
1: 100 \mathrm{on} 0\end{array}$ & $\begin{array}{l}5 \mathrm{ccm} \mathrm{H}_{2} \mathrm{SO}_{4} \text { verdünnt } \\
1 \gg \mathrm{K}_{2} \mathrm{Crr}_{4} \\
\end{array}$ & 3 & negativ & $\begin{array}{l}\text { sehr geringer } \\
\text { Niederschlag }\end{array}$ & Gelbfärbung \\
\hline 15 & $\begin{array}{l}100 \mathrm{ccm} \\
1: 10000\end{array}$ & 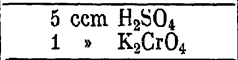 & 3 & do. & do. & $\begin{array}{c}\text { schwacher, } \\
\text { gelb. Niederschl. }\end{array}$ \\
\hline 16 & $\begin{array}{l}100 \mathrm{ccm} \\
1: 10000 \\
\end{array}$ & $\begin{array}{l}5 \mathrm{ccm} \mathrm{H}_{2} \mathrm{SO}_{4} \text { verdünnt } \\
2,5 \gg \mathrm{K}_{2} \mathrm{CrO} \mathrm{O}_{4} \\
\end{array}$ & 3 & do. & $\begin{array}{c}\text { geringer } \\
\text { Niederschlag }\end{array}$ & $\begin{array}{c}\text { schwacher } \\
\text { Niederschlag } \\
\end{array}$ \\
\hline 17 & $\begin{array}{l}100 \mathrm{ccm} \\
1: 10000\end{array}$ & $\begin{array}{l}5 \mathrm{ccm}_{2} \mathrm{H}_{2} \mathrm{SO}_{4} \\
2,5 \gg \mathrm{K}_{2} \mathrm{CrO}_{4} \\
\end{array}$ & 3 & do. & $\begin{array}{c}\text { mäßiger } \\
\text { Niederschlag }\end{array}$ & $\begin{array}{c}\text { Z. starker } \\
\text { Niederschlag } \\
\end{array}$ \\
\hline 18 & $\begin{array}{l}100 \mathrm{ccm} \\
1: 10000\end{array}$ & $\begin{array}{l}5 \mathrm{ccm} \mathrm{H}_{2} \mathrm{SO}_{4} \text { verdünnt } \\
5 \quad * \mathrm{~K}_{2} \mathrm{CrO}_{4} \\
\end{array}$ & 3 & do. & $\begin{array}{c}\text { schwacher } \\
\text { Niederschlag }\end{array}$ & $\begin{array}{c}\text { schwacher } \\
\text { Niederschlag }\end{array}$ \\
\hline 19 & $\begin{array}{l}100 \mathrm{ccm} \\
1: 10000\end{array}$ & $\begin{array}{l}5 \mathrm{ccm} \\
\mathrm{H}_{2} \mathrm{SO}_{4} \\
5 \quad \mathrm{Z}_{2} \mathrm{CrO}_{4} \\
\end{array}$ & 3 & do. & $\begin{array}{c}\text { mäßiger } \\
\text { Niederschlag }\end{array}$ & $\begin{array}{c}\text { starker } \\
\text { Niederschlag }\end{array}$ \\
\hline 20 & $\begin{array}{l}100 \mathrm{ccm} \\
1: 10000 \\
\end{array}$ & $\begin{array}{c}10 \mathrm{ccm} \mathrm{H}_{2} \mathrm{SO}_{4} \\
5 \quad \gg \quad \mathrm{K}_{2} \mathrm{CrO}_{4} \\
\end{array}$ & 2 & do. & do. & $\begin{array}{c}\text { mäBiger } \\
\text { Niederschlag } \\
\end{array}$ \\
\hline 21 & $\begin{array}{l}100 \mathrm{ccm} \\
1: 10000\end{array}$ & $\begin{array}{l}10 \mathrm{c} \cdot \mathrm{cm} \mathrm{H}_{2} \mathrm{SO}_{4} \\
10 * \mathrm{~K}_{2} \mathrm{CrO} \mathrm{Cr}_{4}\end{array}$ & 2 & do. & do. & do. \\
\hline 22 & $\begin{array}{l}100 \mathrm{ccm} \\
1: 20000\end{array}$ & $\begin{array}{l}5 \mathrm{ccm} \mathrm{H}_{2} \mathrm{SO}_{4} \text { verdünnt } \\
5 \gg \mathrm{K}_{2} \mathrm{CrO} \mathrm{r}_{4} \\
\end{array}$ & 3 & do. & $\begin{array}{l}\text { sehr geringer } \\
\text { Niederschlag }\end{array}$ & $\begin{array}{c}\text { schwracher } \\
\text { Niederschlag }\end{array}$ \\
\hline 23 & $\begin{array}{l}100 \mathrm{ccm} \\
1: 20000\end{array}$ & 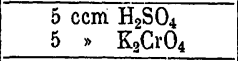 & 3 & do. & $\begin{array}{c}\text { schwacher } \\
\text { Niederschlag }\end{array}$ & $\begin{array}{l}\text { Z. starker } \\
\text { Niederschlag }\end{array}$ \\
\hline 24 & $\begin{array}{l}100 \mathrm{ccm} \\
1: 30000\end{array}$ & 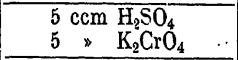 & 2 & do. & do. & do. \\
\hline 25 & $\begin{array}{l}100 \mathrm{ccm} \\
1: 50000\end{array}$ & 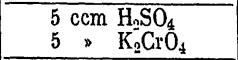 & 2 & do. & $\begin{array}{l}\text { sehr geringer } \\
\text { Niederschlag }\end{array}$ & $\begin{array}{c}\text { Schwacher } \\
\text { Niederschlag } \\
\end{array}$ \\
\hline 26 & $\begin{array}{l}100 \mathrm{ccm} \\
1: 100000\end{array}$ & 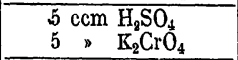 & 2 & do. & do. & \begin{tabular}{|c|} 
sehr schwacher \\
Niederschlag \\
\end{tabular} \\
\hline 27 & $\begin{array}{l}100 \mathrm{ccm} \\
1: 100000\end{array}$ & 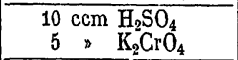 & 2 & do. & $\begin{array}{l}\text { Gorach, nach längerem } \\
\text { steher Niejerschlag }\end{array}$ & Gelbfärbung \\
\hline 28 & $\begin{array}{l}100 \mathrm{ccm} \\
1200000\end{array}$ & $\begin{array}{l}5 \mathrm{ccm} \mathrm{H} \mathrm{H}_{2} \mathrm{SO}_{4} \\
5 \quad \mathrm{~K}_{2} \mathrm{CrO}_{4}\end{array}$ & 2 & do. & do. & $\begin{array}{l}\text { ganz leichte } \\
\text { Gielbfärbung }\end{array}$ \\
\hline
\end{tabular}




\begin{tabular}{|c|c|c|c|c|}
\hline $\begin{array}{l}\text { 1. Destillat } \\
\text { IV. } \\
\text { Silber- } \\
\text { reaktion }\end{array}$ & $\begin{array}{c}\text { Ausfall } \\
\text { im 2. Destillat }\end{array}$ & $\begin{array}{c}\text { Ausfall } \\
\text { im 3. Destillat }\end{array}$ & $\mid \begin{array}{c}\text { Farbe } \\
\text { des } \\
\text { Rück- } \\
\text { stan- } \\
\text { des }\end{array}$ & $\begin{array}{c}\text { Be- } \\
\text { merkungen }\end{array}$ \\
\hline $\begin{array}{l}\text { in der Kälte: braun } \\
\text { b.Erwärmen: Spiegel }\end{array}$ & $\begin{array}{l}\text { Aldehydharzreaktion negativ } \\
\text { II, III, IV schwächer alsim1. Dest. }\end{array}$ & II, III, IV sehr schwach & grün & \\
\hline do. & $\begin{array}{l}\text { I Gelbfärbung } \\
\text { II, III,IV schwächer als im 1.Dest. }\end{array}$ & $\begin{array}{l}\text { I-; II,III,IV schwächer } \\
\text { als im 2. Destillat } \\
\end{array}$ & grün & \\
\hline do. & $\begin{array}{l}\text { I geringer, gelber Niederschlag } \\
\text { II, III,IV schwächer als im 1.Dest. }\end{array}$ & - & grün & \\
\hline do. & do. & - & grün & \begin{tabular}{|l} 
kein Unter- \\
schied gegen 3
\end{tabular} \\
\hline do. & $\begin{array}{l}\text { I sehr schwache Gelbfärbung } \\
\text { II, III, IV positiv, aber schwächer }\end{array}$ & - & grün & \\
\hline do. & do. & - & grün & $\begin{array}{l}\text { stärker als } \\
\text { in } 5\end{array}$ \\
\hline $\begin{array}{l}\text { in der Kälte: nichts } \\
\text { erwärmt: Spiegel }\end{array}$ & $\begin{array}{c}\text { I - } \\
\text { II,III, IV schwächer als im 1. Dest. }\end{array}$ & - & gelb & \\
\hline do. & do. & - & gelb & $\begin{array}{l}\text { stärker als } \\
\text { in } 7\end{array}$ \\
\hline do. & do. & $\begin{array}{l}\text { II, III, IV sehr schwache } \\
\text { Reaktion. }\end{array}$ & gelb & \\
\hline \begin{tabular}{|c|} 
kalt: Bräunung \\
erwärmt: Spiegel \\
\end{tabular} & $\begin{array}{c}\text { I Gelbfärbung } \\
\text { II, III,IV schwächer als im 1. Dest. }\end{array}$ & do. & gelb & \\
\hline do. & do. & - & gelb & \\
\hline do. & do. & - & gelb & $\begin{array}{c}\text { schwächer als } \\
\text { in } 11\end{array}$ \\
\hline do. & $\begin{array}{c}\text { I negativ } \\
\text { II,III,IV schwächer als im 1. Dest. }\end{array}$ & - & gelb & \\
\hline \begin{tabular}{|c|} 
kalt: nichts \\
erhitzt: Braunfärbg.
\end{tabular} & $\begin{array}{c}\text { Inegativ } \\
\text { II-IV Andeut. der Reaktion }\end{array}$ & I-IV negativ & gelb & \\
\hline $\begin{array}{l}\text { kalt: nichts } \\
\text { erhitzt: Spiegel } \\
\end{array}$ & do. & $\begin{array}{c}\text { I negativ } \\
\text { II-IV Reaktion anged. }\end{array}$ & gelb & \\
\hline $\begin{array}{c}\text { kalt: nichts } \\
\text { erhitzt: Braunfärbg. }\end{array}$ & $\begin{array}{c}\text { I negativ } \\
\text { II-IV sehr schwache Reaktion }\end{array}$ & do. & gelb & \\
\hline $\begin{array}{l}\text { kalt: } \text { nichts } \\
\text { erhitzt: Spiegel }\end{array}$ & $\begin{array}{c}\text { I negativ } \\
\text { II-IV schwächer als im 1.Dest. }\end{array}$ & $\begin{array}{l}\text { I negativ } \\
\text { II-IV sehr schwach }\end{array}$ & gellb & \\
\hline do. & do. & do. & gelb & \\
\hline do. & do. & do. & gelb & \\
\hline do. & do. & - & gelb & $\begin{array}{c}\text { schwächer als } \\
\text { in } 19\end{array}$ \\
\hline do. & do. & - & gelb & do. \\
\hline do. & do. & II-IV angedeutet & gelb & \\
\hline do. & do. & do. & gelb & $\begin{array}{l}\text { stärker als } \\
\text { in } 22 \\
\end{array}$ \\
\hline do. & do. & - & gelb & \\
\hline do. & do. & - & gelb & \\
\hline do. & do. & - & gells & \\
\hline \begin{tabular}{|c|} 
kalt: nichts \\
erhitzt: Braunfärbg \\
\end{tabular} & $\begin{array}{l}\text { I und IV negativ } \\
\text { II und III angedeutet }\end{array}$ & - & gelb & $\begin{array}{c}\begin{array}{c}\text { schwächer als } \\
\text { in } 26\end{array} \\
\end{array}$ \\
\hline do. & do. & - & gelb & \\
\hline
\end{tabular}


Die übrigen Reaktionen erwiesen sich auch hier als sehr fein. Die Reaktion mit Nesslers Reagens sowohl wie die Jodoformprobe ergaben noch bei $0,001 \mathrm{ccm}$ Alkoholgehalt im Destillat schwache Niederschläge; auch Silberlösung bildete noch einen Silberspiegel. Da das Ammoniak fast stets reduzierende Substanzen enthielt, so wurde die Probe nur als positiv betrachtet, wenn eine Kontrollprobe entweder ganz negativ ausfiel, oder erst bei erheblich höherer Temperatur Bildung des Silberspiegels eintrat; doch wurde diese Vorsichtsmaßregel bei einem Teil der Versuche nicht beachtet.

Nach den eben angegebenen Methoden wurden nun Organe auf ihren Alkoholgehalt untersucht:

1. Rinderleber, $100 \mathrm{~g}$, einige Stunden nach Tötung des Tieres fein gehackt, mit 300 Aq. dest. abdestilliert. 2 Destillate $\mathrm{zu} 50$ und $100 \mathrm{ccm}$; nach Nicloux titriert (5 bezw. $10 \mathrm{ccm}$ ), schon nach Zusatz von einigen Tropfen $\mathrm{K}_{2} \mathrm{Cr}_{2} \mathrm{O}_{7^{-}}$ Lösung (4,75 g im Liter; diese wurde auch in den übrigen Versuchen verwendet) Gelbfärbung. Rest der Destillale mit $\mathrm{H}_{2} \mathrm{SO}_{4}+\mathrm{K}_{2} \mathrm{CrO}_{4}$-Lösung versetzt. 3 Destillate à $15 \mathrm{ccm}$. Im 1. Destillat: Aldehydharzbildung negativ; $\mathrm{CHJ}_{3}$ : mäßiger Niederschlag; Nesslers Reagens: mäßiger gelber Niederschlag. Silberlösung wird etwas rascher wie Kontrollprobe reduziert; Spiegelbildung. Im 2. und 3. Destillat: schwächerer Ausfall der Reaktionen.

2. Kalbsleber, $100 \mathrm{~g}$, am zweiten Tag nach der Schlachtung, feingehackt, $+200 \mathrm{Aq}$. dest. abdestilliert; 2 Destillate zu 30 und $80 \mathrm{ccm}$, nach Nicloux titriert ( 5 bezw. $10 \mathrm{ccm}$ ), keine Reduktion. Rest $+\mathrm{H}_{2} \mathrm{SO}_{4}+\mathrm{K}_{2} \mathrm{CrO}_{4}$ abdestilliert. 2 Destillate à $15 \mathrm{ccm}$. Im 1. Destillat: Aldehydharzbildung negativ; Nessler: mäßiger gelber Niederschlag; $\mathrm{CHJ}_{3}$-Bildung: mäßiger Niederschlag; Silberlösung: in der Kälte nichts, beim Erwärmen Silberspiegel. Im 2. Destillate: schwächere Reaktionen.

3. Kaninchenleber, $120 \mathrm{~g}, 24$ Stunden post mortem verarbeitet, ergibt in gleicher Weise behandelt gleichen Ausfall der Reaktionen.

4. Rinderleber, $100 \mathrm{~g}, 2$ Tage post mortem verarbeitet, in gleicher Weise behandelt. Titration nach Nicloux : negativ. Die Aldehydreaktionen wie in 2.

5. Rinderleber, $100 \mathrm{~g}$, die gleiche wie in 4 , aber 4 Tage 
liegend, +300 Aq. dest. 2 Destillate $\mathrm{zu} 50$ und $100 \mathrm{ccm}$. 1. Destillat nach Nicloux titriert $(5 \mathrm{ccm}) 0,6 \mathrm{ccm}$ verbraucht. 2. Destillat negativ, enthält also ungefähr $9,5 \mathrm{mg}$ Alkohol. (Konzentr. $=1: 10000-11000$ ). Der Rest der beiden Destillate $+\mathrm{H}_{2} \mathrm{SO}_{4}+\mathrm{K}_{2} \mathrm{CrO}_{4}$ destilliert. 2 Destillate à $15 \mathrm{ccm}$. Im 1. Destillat: Aldehydratharzbildung negativ; Nessler: starker gelber Niederschlag; $\mathrm{CHJ}_{3}$ : ziemlich starker Niederschlag; Silberlösung: in der Kälte nichts, bei leichtem Erwärmen Spiegelbildung. Im 2. Destillat schwächere Reaktionen.

6. Muskelfleisch vom Rind, $200 \mathrm{~g}$, 4 Tage nach der Tötung feingehackt +400 Aq. dest. 2 Destillate von 80 und $120 \mathrm{ccm}$. 1. Destillat nach Nicloux titriert $(10 \mathrm{ccm}), 1,8 \mathrm{ccm} \mathrm{K} \mathrm{Cr}_{2} \mathrm{O}_{7}$ Lösung verbraucht. 2. Destillat: für $10 \mathrm{ccm} 0,5 \mathrm{ccm}$ Chromlösung verbraucht. Alkoholgehalt $=0,04 \mathrm{ccm}$ (Konzentration $=1: 4500$ ). Rest der Destillate $+\mathrm{H}_{2} \mathrm{SO}_{4}+\mathrm{K}_{2} \mathrm{CrO}_{4}$ destilliert. 2 Destillate. Im 1. Destillat: Aldehydharzbildung vorhanden (schwacher Niederschlag); Nessler: dicker, bald grau werdender Niederschlag; $\mathrm{CHJ}_{3}$ : starker Niederschlag; Silber: in der Kälte Bräunung, beigeringem Erwärmen Silberspiegel. Im 2. Destillat alle Reaktionen schwächer.

7. $200 \mathrm{~g}$ desselben Fleisches, 7 Tage gelegen, $+600 \mathrm{ccm}$ Aq. dest. 2 Destillate zu 80 und $220 \mathrm{ccm}$. Nach Nicloux titriert: vom 1 . Destillat $10 \mathrm{ccm}$, verbraucht $3,4 \mathrm{ccm} \mathrm{K}_{2} \mathrm{Cr}_{2} \mathrm{O}_{7}$ Lösung, vom 2. Destillat $10 \mathrm{ccm}, 0,5 \mathrm{~cm} \mathrm{~K} \mathrm{Kr}_{2} \mathrm{O}_{7}$-Lösung. Alkoholgehalt der Destillate 0,06 ccm (Konzentration 1: 3300). Der Rest der Destillate mit $\mathrm{H}_{2} \mathrm{SO}_{4}+\mathrm{K}_{2} \mathrm{CrO}_{4}$ destilliert, 4 Destillate zu $15 \mathrm{ccm}$. Die Aldehydreaktionen sind wie in 6, vielleicht etwas stärker, im 3. und 4. Destillate ist die $\mathrm{NaOH}-$ Reaktion negativ.

8. Rinderleber, $125 \mathrm{~g}, 2$ Tage alt, mit $300 \mathrm{Aq}$. zerhackt, 14 Stunden im Brutschrank, Geruch unangenehm, aber nicht direkt faulig. 2 Destillate zu 50 und $100 \mathrm{ccm}$. Von $5 \mathrm{ccm}$ des ersten Destillates werden $4,2 \mathrm{~cm} \mathrm{~K}_{2} \mathrm{Cr}_{2} \mathrm{O}_{7}$-Lösung reduziert, von $10 \mathrm{ccm}$ des zweiten $0,4 \mathrm{ccm} \mathrm{K}_{2} \mathrm{Cr}_{2} \mathrm{O}_{7}$-Lösung. Alkoholgehalt= $0,072 \mathrm{ccm}$ (Konzentration $1: 1700$ ). Rest der beiden Destillate $+\mathrm{H}_{2} \mathrm{SO}_{4}+\mathrm{K}_{4} \mathrm{CrO}_{4}$ destilliert. 3 Destillate à $15 \mathrm{ccm}$. Alle Aldehydreaktionen fallen stark positiv aus; im 1. Destillate am stärksten, im 3. ist die $\mathrm{NaOH}$-Reaktion negativ.

9. Gleiche Menge Rinderleber wie 8 , nach Zusatz von $1 \mathrm{~g}$ 
Dextrose gleich behandelt. 1. Destillat reduziert (5 ccm) $4,6 \mathrm{ccm}$ $\mathrm{K}_{2} \mathrm{Cr}_{2} \mathrm{O}_{7}$-Lösung, 2. Destillat $(10 \mathrm{ccm})$ 0,6 ccm. Alkoholgehalt also $=0,081 \mathrm{ccm}$ (Konzentration 1:1500). Die weitere Behandlung wie in 8, gleicher Ausfall der Aldehydreaktionen.

10. Rinderleber, $100 \mathrm{~g}$, mit 300 Aq. 2 Tage im Brutofen stehend, nach 2 Tagen stinkende Fäulnis, destilliert. 2 Destillate $\mathrm{zu} 50$ und $100 \mathrm{ccm}$. Titration nach Nicloux unmöglich, da nach Zusatz von $\mathrm{H}_{2} \mathrm{SO}_{4}$ eine gelbrötliche Eigenfärbung der Flüssigkeit eintritt, die den Farbenumschlag nicht erkennen läßt. Reste der Destillate $+\mathrm{K}_{2} \mathrm{CrO}_{4}+\mathrm{H}_{2} \mathrm{SO}_{4}$ destilliert; 3 Destillate à $15 \mathrm{ccm}$. Im 1. sind alle Reaktionen sehr stark positiv, im 3. die $\mathrm{NaOH}-$ Reaktion negativ.

11. Rinderleber, $100 \mathrm{~g},+1 \mathrm{~g}$ Dextrose, sonst wie 10 behandelt. Titration aus gleichem Grunde unmöglich. Rest ebenfalls mit $\mathrm{H}_{2} \mathrm{SO}_{4}+\mathrm{K}_{2} \mathrm{CrO}_{4}$ destilliert; Ausfall der Reaktionen in den 3 Destillaten wie in 10.

Bei diesen nicht lebensfrischen Organen fand sich also stets Alkohol, in den Fällen, in denen die Organe noch nicht lange gelegen hatten (1-4), war er quantitativ nicht zu bestimmen; in den übrigen Fällen war auch seine Quantität bestimmbar, sie nahm mit der Dauer des Liegens der Organe vor der Verarbeitung bezw. mit der Intensität der Fäulnis zu. Ob der Zusatz von Dextrose seine Menge vermehrte, läßt sich nicht mit Sicherheit sagen, denn im Versuch 9 ist die Vermehrung gegen Versuch 8 nur unerheblich; im Versuche 10 und 11 aber war die Ausführung der Titration unmöglich. Diese Versuche zeigen mithin, daß in tierischen Organen unter bakterieller Einwirkung Alkohol entsteht. Wenn auch in einem Falle (1) nahezu lebensfrisches Material verwendet wurde, so war es doch fraglich, ob sich der Alkohol auch normalerweise in lebensfrischen Organen fände. Um dies festzustellen, wurden Leber und Muskelfleisch von Kaninchen sofort nach der Tötung verarbeitet.

12. Muskelfleisch, $150 \mathrm{~g}$, feingehackt mit $300 \mathrm{Aq}$. destilliert, 2 Destillate zu 60 und $100 \mathrm{ccm}$. Titriert nach Nicloux, schon nach 2-3 Tropfen Zusatz Gelbfärbung. Die beiden Destillate $+\mathrm{H}_{2} \mathrm{SO}_{4}+\mathrm{K}_{2} \mathrm{CrO}_{4}$ destilliert; 3 Destillate à $15 \mathrm{ccm}$. Im 1. Destillat: Aldehydharzbildung negativ. Nessler: mäßiger gelber 
Niederschlag; $\mathrm{CHJ}_{3}$ : mäßiger Niederschlag, Silber: in der Kälte nichts, beim Erhitzen Silberspiegel. Im 2. und 3. Destillate dieselben Reaktionen, aber schwächer.

13. Muskelfleisch, $420 \mathrm{~g}$, mit $600 \mathrm{Aq}$. destilliert. In den beiden Destillaten zu 100 und $200 \mathrm{ccm}$ tritt bei Titrat nach Nicloux schon bei einigen Tropfen Zusatz Gelbfärbung auf. Rest mit $\mathrm{H}_{2} \mathrm{SO}_{4}+\mathrm{K}_{2} \mathrm{CrO}_{4}$ destilliert. 3 Destillate à $15 \mathrm{ccm}$ geben die gleichen Reaktionen wie in 12 , nur etwas intensiver.

14. Muskelfleisch $320 \mathrm{~g},+600$ Aq. destilliert. 2 Destillate zu 100 und $200 \mathrm{ccm} .10 \mathrm{ccm}$ des 1 . Destillates nach Nicloux titriert, 1,7 ccm $\mathrm{K}_{2} \mathrm{Cr}_{2} \mathrm{O}_{7}$-Lösung verbraucht, im zweiten schon nach einigen Tropfen Gelbfärbung. Alkoholgehalt $=0,027 \mathrm{ccm}$ (Konzentration $=1: 11000-12000$ ). Rest der beiden Destillate mit $\mathrm{H}_{2} \mathrm{SO}_{4}+\mathrm{K}_{2} \mathrm{CrO}_{4}$ destilliert. 4 Destillate à $15 \mathrm{ccm}$. Im ersten Destillat beimErhitzen mit $\mathrm{NaOH}$ Aldehydharzbildung mit geringem Niederschlag. $\mathrm{CHJ}_{3}$ : starker Niederschlag; Nestler: starker, bald grauwerdender Niederschlag; Silber: beimErwärmenSilberspiegel. Im 2. bis 4. Destillat Aldehydharzbildung negativ; die übrigen Proben positiv.

15. Muskelfleisch, $460 \mathrm{~g}$, eines Kaninchens, das am Vor- und Nachmittag des der Tötung vorangehenden Tages je $15 \mathrm{~g}$ Dextrose mit Schlundsonde zugeführt erhalten hat, außerdem $30 \mathrm{~g}$ Dextrose zur Nahrung zugemischt erhielt, mit 600 Aq. destilliert. 2 Destillate von 100 und $200 \mathrm{ccm}$. Im 1. Destillat für $10 \mathrm{ccm}$ bei der Titration $0,8 \mathrm{ccm} \mathrm{K}_{2} \mathrm{Cr}_{2} \mathrm{O}_{7}$-Lösung verbraucht, im 2. nach einigen Tropfen schon Gelbfärbung. Alkoholgehalt $0,013 \mathrm{ccm}$ (Konzentration $1: 33000$ ). Weitere Behandlung wie sonst. Ausfall der Reaktionen wie in 13.

16. Leber, $105 \mathrm{~g},+200$ Aq. destilliert. 2 Destillate zu 30 und $70 \mathrm{ccm}$. Titration bei beiden schon nach Zusatz weniger Tropfen $\mathrm{K}_{2} \mathrm{Cr}_{2} \mathrm{O}_{7}$-Lösung Gelbfärbung. Nach Destillation mit $\mathrm{K}_{2} \mathrm{CrO}_{4}+\mathrm{H}_{2} \mathrm{SO}_{4}$ fallen Reaktionen aus wie bei 12 .

17. Leber, $96 \mathrm{~g}$, von Kaninchen mit Dextrosefütterung +200 Aq. dest. (s. 15) destilliert. 2 Destillate zu 30 und $70 \mathrm{ccm}$. Titration der beiden Destillate nach Nicloux nach wenigen Tropfen Zusatz Gelbfärbung. Weitere Behandlung und Ausfall der Reaktionen wie bei 12.

Hoppe-Seyler's Zeitschrift f. physiol. Chemie. XLI. 34 
18. Leber, $100 \mathrm{~g}$, von Kaninchen, das (unter Wasserzufuhr) 7 Tage vor der Tötung gehungert hat, +200 Aq. destilliert. 2 Destillate zu 30 und $70 \mathrm{ccm}$. Weitere Behandlung und Ausfall der Reaktionen wie in 17.

Auch in diesen ganz lebensfrischen Organen also ließ sich der Alkohol qualitativ stets nachweisen; in zwei Versuchen (14 und 15) auch quantitativ. Im Versuch 14 betrug die Konzentration des Alkohols in dem Gewebe $1: 11000-12000$, im Versuche 15 1:33000. In diesem letzten Versuche hatte das Kaninchen vor der Tötung größere Dextrosemengen zugeführt erhalten, im Versuch 14 hatte es gewöhnliche Kohlfütterung. In den übrigen Fällen ließ sich die Konzentration des Alkohols nach der Intensität der Reaktionen annäherungsweise auf 1:50000-70000 schätzen, auch im Versuche 18 (Organ von Hungerkaninchen). Da auch im Versuch 17 (Kaninchen mit Dextrosefütterung) die Intensität der Reaktionen durchaus nicht stärker war als in den übrigen Fällen, so erscheint es nicht sehr wahrscheinlich, daß die Ernährungsweise einen bestimmenden Einfluß auf die Konzentration des Alkohols bezw. seine Menge ausübt.

$\mathrm{Da}$ der Alkohol stets als Aldehyd nachgewiesen wurde, so ließe sich der Einwand erheben, daß in den Organen nicht Alkohol, sondern Aldehyd vorhanden sei. Einmal aber würde der präformierte Aldehyd durch Chromsäure fast sicher weiter oxydiert werden und somit dem Nachweis entgehen; dann aber läßt es sich auch auf andere Weise zeigen, daß es sich nicht um präformierten Aldehyd handeln kann. Es wurden nämlich von zwei Tage liegender Hundeleber, von lebensfrischem Kaninchenmuskelfleisch und ebensolcher Kaninchenleber durch Destillation mit Wasser Destillate gewonnen, die dann folgendermaßen behandelt wurden. Jedes Destillat wurde in zwei Hälften geteilt, die eine Hälfte unter Zusatz von $\mathrm{H}_{2} \mathrm{SO}_{4}+\mathrm{K}_{2} \mathrm{CrO}_{4}$, die andere nur nach schwachem Ansäuren mit $\mathrm{HCl}$ destilliert, von beiden Portionen gleiche Mengen Destillate (3 Destillate à $15 \mathrm{ccm}$ ) aufgefangen und die Intensität der Aldehydreaktionen in den entsprechenden Destillaten verglichen. Dabei zeigte es sich, daß in der mit Chromsäure behandelten Portion im ersten Destillat 
ziemlich starke Aldehydreaktionen auftraten, die schwächer auch noch in den beiden anderen Destillaten erhalten wurden, während in der nur schwach angesäuerten Portion im ersten Destillat nur die $\mathrm{CHJ}_{3}$-Probe und die Reaktion mit Nesslers Reagens ganz schwach positiv ausfiel, in den anderen Destillaten alle Reaktionen negativ verliefen. Wenn es daher auch nicht ausgeschlossen erscheint, daß ganz geringe Spuren Aldehyd präformiert vorhanden sind, so ist doch mit Sicherheit zu schließen, daß die Hauptmenge des gewonnenen Aldehyds erst durch die Oxydation mittels Chromsäure entstanden ist, und als Körper, aus dem der Aldehyd entstanden sein kann, kommt nach unseren bisherigen Kenntnissen wohl nur der Alkohol in Betracht.

Von Interesse war auch die Frage, ob sich etwa bei der Autolyse der Organe Alkohol bilde. Will doch in letzter Zeit Stoklasa ${ }^{15}$ ) und seine Schüler unter der Einwirkung des glykolytischen Fermentes der Gewebe bei Abschluß von Sauerstoff das Auftreten großer Mengen Alkohols beobachtet haben. Diese Beobachtungen sind aber von verschiedenen Seiten bestritten worden. So hat Blumenthal ${ }^{16}$ ) bei der Glykolyse mit Sicherheit das Auftreten von Alkohol nicht feststellen können, ebensowenig Arnheim und Rosenbaum;17) Umber ${ }^{18}$ ) bezweifelt sogar überhaupt die Wirksamkeit eines glykolytischen Fermentes und meint, daß die Produkte, die andere Autoren der Glykolyse zuschreiben, nichts weiter als bakterielle Zersetzungsprodukte sind.

Da die aseptische Autolyse infolge der Schwierigkeit ihrer Durchführung wenig aussichtsreich erschien, so wurde in den folgenden Versuchen die antiseptische Autolyse unter Zusatz von Toluol $(2-5 \%)$ gewählt. $\left.{ }^{1}\right)$ In allen Fällen wurden lebensfrische Organe von Kaninchen verwendet.

19. Muskelfleisch von Kaninchen (vom gleichen Tier wie in Versuch 12), $150 \mathrm{~g}+300$ Aq. $+10 \mathrm{~g}$ Toluol, unter häufigem Umschütteln 6 Tage im Brutschrank stehend; darauf ohne Zusatz destilliert. 2 Destillate zu 50 und $100 \mathrm{ccm}$. Titration nach Nicloux, in beiden Destillaten schon nach einigen Tropfen

1) Von der Anwendung von Chloroform wurde abgesehen, da hierdurch Komplikationen für den Nachweis entstehen. 
$\mathrm{K}_{2} \mathrm{Cr}_{2} \mathrm{O}_{7}$-Zusatz Gelbfärbung. Rest der Destillate $+\mathrm{K}_{2} \mathrm{CrO}_{4}+$ $\mathrm{H}_{2} \mathrm{SO}_{4}$ destilliert. 3 Destillate à $15 \mathrm{ccm}$. Im ersten Destillat: $\mathrm{NaOH}-R e a k t i o n$ negativ; Nessler: mäßiger, gelber Niederschlag; $\mathrm{CHJ}_{3}$ : mäßiger Niederschlag; Silber: bei mäßigem Erwärmen Silberspiegel. 2 Gelatineröhrchen geimpft, nach 2 Tagen steril und solange die Beobachtung fortgesetzt wurde (ca. 14 Tage).

20. Muskelfleisch, $350 \mathrm{~g},+500$ Aq. $+10 \mathrm{~g}$ Toluol, 4 Tage im Brutschrank, Kulturen nach 2 Tagen steril. Behandlung und Reaktionen wie in 19.

21. Muskelfleisch, von Hungerkaninchen (s. 18), $210 \mathrm{~g}+$ 300 Aq. $+10 \mathrm{ccm}$ Toluol, 4 Tage stehend, destilliert; 2 Destillate $\mathrm{zu} 50$ und $100 \mathrm{ccm}$. Im 1. Destillat zu $5 \mathrm{ccm}$ verbraucht $1,6 \mathrm{ccm} \mathrm{K} \mathrm{Kr}_{2} \mathrm{O}_{7}$-Lösung, im 2. Destillat zu $10 \mathrm{ccm} \mathrm{1,1} \mathrm{ccm.}$ Alkoholgehalt $42 \mathrm{mg}$ (Konzentration $1: 5000)$. Rest des Destillats $+\mathrm{H}_{2} \mathrm{SO}_{4}+\mathrm{K}_{2} \mathrm{CrO}_{4}$ destilliert. 3 Destillate à $15 \mathrm{ccm}$. Im ersten Destillat: NaOH-Reaktion: schwach gelber Niederschlag; Nessle rs Reagens: dicker, bald grauwerdender Niederschlag; $\mathrm{CHJ}_{3}$ : sehr starker Niederschlag; Silber: kalt Bräunung, bei geringem Erwärmen Spiegelbildung. In den andern Destillaten schwächere Reaktionen, im 3. die NaOH-Reaktion negativ. Impfung von Gelatineplatten leider versäumt.

22. Kaninchenleber, $150 \mathrm{~g}+300 \mathrm{Aq} .+10 \mathrm{ccm}$ Toluol 4 Tage im Brutschrank; gleiche Behandlung und gleiche Reaktionen wie in 19 . Gelatineröhrchen steril.

23. Kaninchenleber, $100 \mathrm{~g},+300 \mathrm{Aq} .+10 \mathrm{ccm}$ Toluol, 3 Tage im Brutschrank. Behandlung und Reaktionen wie in 19. Gelatineröhrchen steril.

24. Kaninchenleber, $105 \mathrm{~g},+300$ Aq. $+10 \mathrm{ccm}$ Toluol, 3 Tage im Brutschrank stehend. Behandlung und Reaktionen wie in 19. Gelatineröhrchen steril.

25. Kaninchenleber, $145 \mathrm{~g},+300 \mathrm{Aq} .+10 \mathrm{ccm}$ Toluol $+1 \mathrm{~g}$ Dextrose, 3 Tage im Brutschrank stehend, sonst Behandlung und Reaktionen wie in 19. Gelatineröhrchen steril.

Es ergab sich also bei den autolytischen Versuchen, daß in allen den Fällen, in denen durch Sterilbleiben der Gelatineröhrchen bakterielle Zersetzung ausgeschlossen werden konnte, irgendwelche merkbare Zunahme des Alkoholgehaltes nicht ein- 
trat, auch nicht nach Zusatz von Dextrose (Versuch 25). Daß das Toluol, welches ja Alkohol auch sehr leicht löst, keine wesentlichen Mengen Alkohols in sich aufnimmt und somit keine erheblichen Fehlerquellen bei den Versuchen bietet, wurde in mehreren Versuchen festgestellt. Es wurde in vier Fällen das Toluol (ca. $10 \mathrm{ccm}$ ) vom Destillat im Scheidetrichter abgetrennt und dann fünfmal hintereinander mit je $10 \mathrm{ccm}$ destillierten Wassers geschüttelt, das Wasser abgetrennt und mit $\mathrm{H}_{2} \mathrm{SO}_{4}$ $+\mathrm{K}_{2} \mathrm{CrO}_{4}$ destilliert. Im ersten Destillat (zu $10 \mathrm{ccm}$ ) waren in drei Fällen Andeutungen von Aldehydreaktionen, im vierten Falle auch diese nicht einmal vorhanden; im zweiten Destillat (zu $10 \mathrm{ccm}$ ) waren die Reaktionen durchweg negativ. Auch die Titration einer Alkohollösung von 1:5000 ließ nur ganz geringe Unterschiede vor und nach dem Schütteln mit Toluol erkennen. Das Ausschütteln dieses Toluols mit Wasser und nachfolgende Destillation mit $\mathrm{H}_{2} \mathrm{SO}_{4}+\mathrm{K}_{2} \mathrm{CrO}_{4}$ ergab ebenfalls Andeutungen der Aldehydreaktionen. Es wird daher also höchstens ein sehr kleiner Bruchteil des Alkohols vom Toluol aufgenommen, und die Konservierung der autolytischen Flüssigkeiten mit Toluol bedingt somit sicher keinen wesentlichen Fehler. In dem einzigen Falle (Versuch 21), in dem ein starker Alkoholgehalt sich fand, war leider gerade die Impfung von Gelatineröhrchen verabsäumt worden. Nach den übereinstimmenden Befunden der übrigen Untersuchungen muß daher hier wohl eine Alkoholbildung unter bakterieller Zersetzung angenommen werden.

Somit kann man als Resultat der vorliegenden Untersuchung wohl feststellen, daß sich Alkohol in geringen Mengen präformiert in den Geweben findet, und daß bei der Autolyse seine Menge nicht merklich zunimmt, wohl aber bei der bakteriellen Zersetzung. Die Frage nach der Abstammung des Alkohols in den Geweben läßt sich nicht bestimmt beantworten. Da Arnheim und Rosenbaum in ihren Versuchen, bei denen sie verschiedene Gewebe mit Zuckerlösung digerierten, wohl eine Zerstörung des Zuckers feststellen konnten, eine Alkoholbildung aber nur in denjenigen Versuchen, in denen es nicht gelungen war, Bakterienwirkung auszuschließen, so kommt zur Erklärung des spurenweisen Alkoholgehaltes in den Geweben die Entstehung an Ort 
und Stelle wohl kaum in Betracht. Das Wahrscheinlichste ist, daß der Alkohol von der Zersetzung der Kohlehydrate im Magendarmkanal durch Hefepilze oder Bakterien abstammt. Bei der Fäulnis außerhalb des Körpers kommen als Quelle für ihn wohl auch die Kohlehydrate am ehesten in Betracht.

Herrn Geheimrat Salkowski sage ich zum Schluß für die Anregung $\mathrm{zu}$ dieser Arbeit und seine gütige Unterstützung bei ihrer Ausführung meinen ergebensten Dank.

\section{Literatur.}

1. Hudson Ford, Über das normale Vorkommen von Alkohol im Blut. Journal of the Elliott Society of Nat. History, Vol. I. Art. II, pag. 43-99. Auszug in Schmidts Jahrbüchern, Bd. 112, 1861.

2. A. Béchamp, Sur la fermentation alcoolique et acétique spontanée du foie et sur l'alcool physiologique de l'urine humaine. Comptes rendus de l'Académie des sciences, Bd. 75, 1872, S. 1830.

3. A. Béchamp, Sur l'alcool et l'acide acétique normaux du lait comme produits de la fonction des microzymas. Compt. rend. 1873, Bd. 76, S. 836.

4. J. Béchamp, Sur la présence de l'alcool dans les tissus animaux pendant la vie et après la mort dans le cas de putréfaction au point de vue physiologique et toxicologique. Compt. rend., Bd. 89, 1879, S. 573, und Ann. de chimie et de physique, 1880, S. 406.

5. Rajewsky, Über das Vorkommen von Alkohol im Organismus. Pflügers Archiv, Bd. 11, 1875, S. 122-128.

6. Vitali, Alkohol bei der fauligen Gährung von Eiereiweiß. Annal. di chim. e di farmac., 4. Serie, Bd. V, S. 113.

7. Albertoni, Über Bildung und Vorkommen von Alkohol und Aldehyd im Organismus. Ann. di chim. e di farmac., 4. Serie, Bd. 6, S. $250-258$.

8. M. Nicloux, Dosage de l'alcool éthylique. Comptes rendues de la Société de biologie, 10e série, Bd. 3, 1896, S. 841.

9. M. Nicloux, Recherches expérimentales sur l'élimination de l'alcool dans l'organisme. Paris, 1900, b. Doin.

10. Bodländer, Die Ausscheidung aufgenommenen Weingeistes aus dem Körper. Pflügers Archiv, Bd. 32, 1883, S. 398.

11. Lieben, Alkohol geht in den Harn über. Annalen für Chemie und Pharmacie, VII. Supplementb. 1870, S. 236.

12. Béhal und François, Dosage de l'alcool.

13. Straßmann, Untersuchungen über den Nährwert und die Ausscheidung des Alkohols. Pflügers Archiv, Bd. 49, 1891, S. 315. 
14. Norris und Benedict, Die Bestimmung kleiner Mengen von Alkohol. Journal of Americ. Chem. Society, Bd. 20, S. 293. Referat darüber in Chemisches Zentralblatt, 1898, I, S. 1069.

15. Stoklasa, Die glykolytischen Enzyme im tierischen Gewebe. Deutsche medizinische Wochenschrift, 1904, Nr. 6, S. 198 (mit Literaturangaben).

16. F. Blumenthal, Über das glykolytische Ferment. Vortrag im Verein für innere Medizin (Berlin), 16. Nov. 1903. Referat in Berliner klinische Wochenschrift, 1903, Nr. 48.

17. Arnheim und Rosenbaum, Ein Beitrag zur Frage der Zuckerzerstörung im Tierkörper durch Fermentwirkung (Glykolyse). Diese Zeitschrift, Bd. XL, S. 220 (1904).

18. Umber, Zur Lehre von der Glykolyse. Zeitschrift f. klinische Medizin, Bd. 39, 1900, S. 12. 